\title{
A Case of Paraneoplastic Syndrome
}

\author{
Masashi Ohe \\ Department of General Medicine, JCHO Hokkaido Hospital, Sapporo, Japan
}

\begin{abstract}
A 73-year-old woman was admitted with fever, arthralgia, and swelling of the hands and feet. She was diagnosed with remitting seronegative symmetrical synovitis with pitting edema (RS3PE) accompanied by breast carcinoma, a type of paraneoplastic syndrome. Treatment with acetaminophen and diclofenac sodium for fever and arthralgia proved minimally effective. She experienced great difficulty in walking and grasping smoothly due to edema; therefore, clarithromycin (CAM) was added in expectation of its anti-inflammatory effects. About 10 days after starting CAM treatment, pitting edema improved considerably. Fever and arthralgia did not improve satisfactorily after resection of breast carcinoma, and thus she was successfully treated with low-dose prednisolone (PSL). This case suggests that other than surgical resection of the neoplasm, CAM and PSL treatment for RS3PE associated with neoplastic disease may be effective.
\end{abstract}

Keywords: Clarithromycin; Prednisolone; Breast neoplasms

\section{INTRODUCTION}

Remitting seronegative symmetrical synovitis with pitting ede$\mathrm{ma}$ (RS3PE) is an inflammatory syndrome characterized by symmetrical sinovitis with pitting edema of the dorsa of the hands and/ or feet. Glucocorticoid (GC) remains the mainstay of treatment for RS3PE not associated with neoplastic disease. GC therapy usually dramatically improves the clinical picture, but is known to be of limited use for RS3PE associated with neoplastic disease. Macrolide antibiotics such as erythromycin and clarithromycin (CAM) show not only antibacterial activity, but also anti-inflammatory effects. In terms of anti-inflammatory effects for rheumatic diseases, successful macrolide treatment of rheumatoid arthritis has been reported [1]. We report herein a case of RS3PE associated with breast carcinoma, in which pitting edema and continuous fever/arthralgia after resection of the carcinoma were successfully treated using CAM and prednisolone (PSL), respectively.

\section{CASE REPORT}

A 73-year-old woman was admitted with a 2-week history of fe- ver, acute arthralgia, and edema of both the hands and feet. She had a 4-year history of diabetes mellitus (DM), but no significant family history of relevant illnesses, including rheumatic disease. Physical examination revealed slightly tender articular swellings in bilateral wrist, hand, knee and foot joints, with marked pitting edema on bilateral dorsa of the hands and feet (Fig. 1A). No symptoms or signs of collagen disease, including exanthema, muscle weakness, and Raynaud's phenomenon, were evident. Laboratory findings (Table 1) were as follows: white blood cell count, $8,090 / \mu \mathrm{L}$; aspartate aminotransferase, $16 \mathrm{IU} / \mathrm{L}$; alanine aminotransferase, $7 \mathrm{IU} / \mathrm{L}$; blood urea nitrogen, $7.8 \mathrm{mg} / \mathrm{dL}$; creatinine, $0.32 \mathrm{mg} / \mathrm{dL}$; total protein, 7.5 $\mathrm{g} / \mathrm{dL}$; albumin, $2.7 \mathrm{~g} / \mathrm{dL}$; and C-reactive protein (CRP), $18.5 \mathrm{mg} / \mathrm{dL}$. Fasting blood sugar and hemoglobin Alc levels were $133 \mathrm{mg} / \mathrm{dL}$ and 7.0\%, respectively. Serological tests showed the following: immunoglobulin (Ig)G, 1,873 mg/dL; IgM, 90 mg/dL; IgA, 522 mg/ $\mathrm{dL}$; rheumatoid factor (RF), <15.0 IU/L; $\mathrm{C}_{3}, 164 \mathrm{mg} / \mathrm{dL}$; and $\mathrm{C}_{4}, 41$ $\mathrm{mg} / \mathrm{dL}$. Matrix metalloproteinase 3 level was $344.8 \mathrm{ng} / \mathrm{mL}$. No immune complexes (IC) were detected using the $\mathrm{C}_{\mathrm{lq}}$-binding assay. Anti-nuclear antibody titer was $<40$. Negative results were also obtained for all other autoimmune antibodies, including anti-SSA, anti-SSB, anti-DNA, anti-RNP, anti-Sm, anti-cardiolipin (anti-CL),
Correspondence to: Masashi Ohe

Department of General Medicine, JCHO Hokkaido Hospital, 1-8-3-18 Nakanoshima, Toyohira-ku, Sapporo 062-8618, Japan

Tel: +81-11-831-5151, Fax: +81-11-821-3851, E-mail: masshi@isis.ocn.ne.jp

Received: Jun. 12, 2014 / Accepted after revision: Dec. 26, 2014
(C) 2014 Soonchunhyang Medical Research Institute This is an Open Access article distributed under the terms of the Creative Commons Attribution Non-Commercial License (http://creativecommons.org/licenses/by-nc/3.0/). 

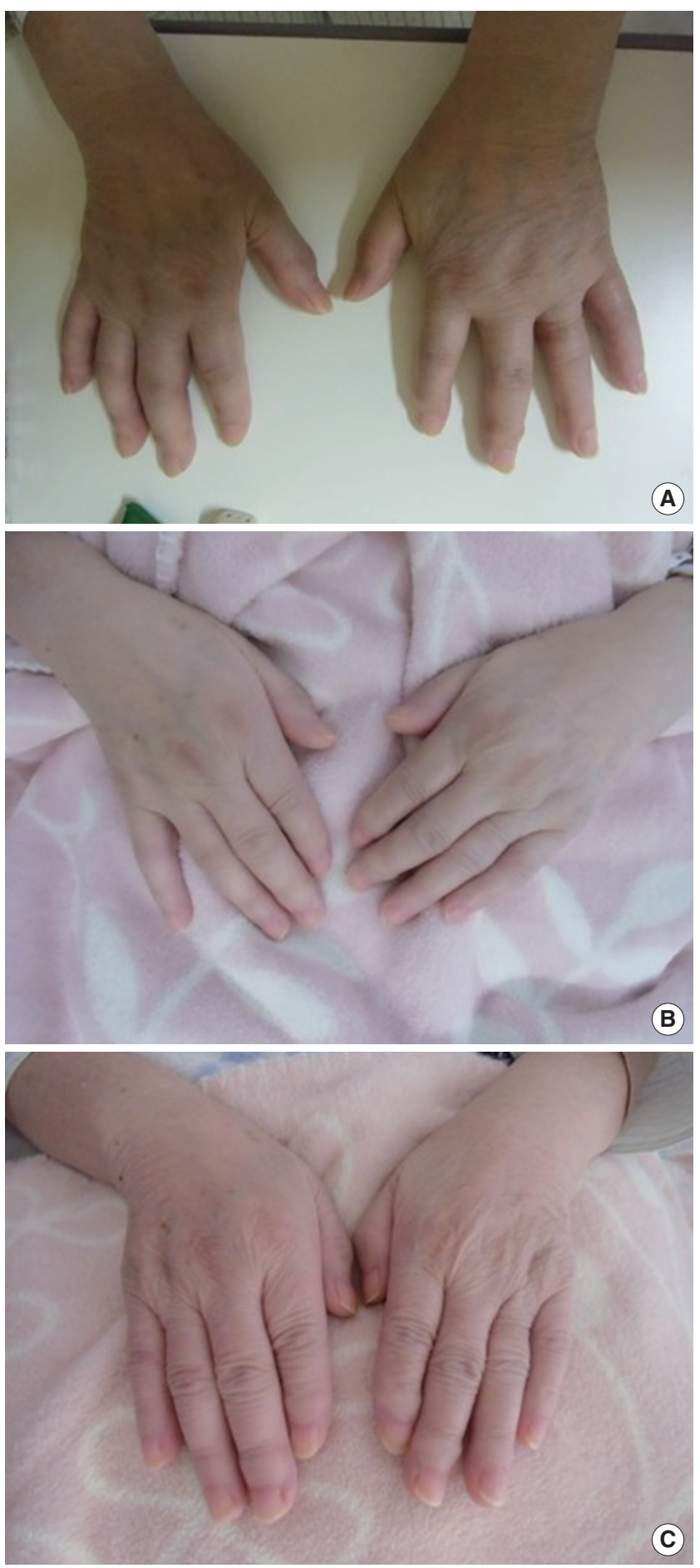

Fig. 1. (A) Bilateral dorsa of the hands and wrists shows marked pitting edema on admission. (B) Marked pitting edema shows considerable improvement 10 days after starting clarithromycin treatment. (C) Marked pitting edema shows complete improvement 2 weeks after starting prednisolone treatment.
Table 1. Laboratory data on admission

\begin{tabular}{|c|c|c|}
\hline Variable & Value & Normal range \\
\hline White blood cell count $(/ \mu \mathrm{L})$ & 8,090 & $4,000-9,000$ \\
\hline Neutrophil (\%) & 66.3 & $44.0-77.0$ \\
\hline Lymphoid (\%) & 26.7 & $20.0-55.0$ \\
\hline 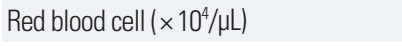 & 381 & $380-480$ \\
\hline Hemoglobin (g/dL) & 9.5 & $11.0-16.0$ \\
\hline Platelet count $\left(\times 10^{4} / \mu \mathrm{L}\right)$ & 53.2 & $15.0-44.0$ \\
\hline Total protein (g/dL) & 7.5 & $6.5-8.0$ \\
\hline Albumin (g/dL) & 2.7 & $>4.0$ \\
\hline $\mathrm{Na}(\mathrm{mEq} / \mathrm{L})$ & 140 & $138-145$ \\
\hline $\mathrm{Cl}(\mathrm{mEq} / \mathrm{L})$ & 100 & $99-108$ \\
\hline $\mathrm{K}(\mathrm{mEq} / \mathrm{L})$ & 3.6 & $3.4-4.7$ \\
\hline Blood urea nitrogen (mEq/L) & 7.8 & 7.0-20.9 \\
\hline Creatinine (mEq/L) & 0.32 & $<0.89$ \\
\hline Uric acid (mEq/L) & 4.0 & $<7.0$ \\
\hline Aspartate aminotransferase (U/L) & 16 & $<35$ \\
\hline Alanine aminotransferase (U/L) & 7 & $<35$ \\
\hline Alkaline phosphatase (U/L) & 202 & $<339$ \\
\hline Y-Glutamyl transpeptidase (U/L) & 15 & $<55$ \\
\hline Fasting blood sugar (mg/dL) & 133 & $60-109$ \\
\hline Hemoglobin A1c (\%) & 7.0 & $4.6-6.2$ \\
\hline Carcinoembryonic antigen (ng/mL) & 0.7 & $<5.0$ \\
\hline Carbohydrate antigen 19-9 (U/mL) & 5.6 & $<37.0$ \\
\hline Carbohydrate antigen 15-5 (U/mL) & 5.3 & $<27.0$ \\
\hline Free T4 (ng/dL) & 1.260 & $0.90-1.70$ \\
\hline C-reactive protein (mg/dL) & 18.5 & $<0.3$ \\
\hline $\lg \mathrm{g}(\mathrm{mg} / \mathrm{dL})$ & 1,873 & $870-1,700$ \\
\hline $\operatorname{lgM}(\mathrm{mg} / \mathrm{dL})$ & 90 & $35-220$ \\
\hline $\operatorname{lgA}(\mathrm{mg} / \mathrm{dL})$ & 522 & $110-440$ \\
\hline Rheumatoid factor (U/mL) & $<15$ & $<15$ \\
\hline Matrix metalloproteinase-3 (ng/mL) & 344.8 & $17.3-59.7$ \\
\hline Anti-nuclear Ab & $<40$ & $<80$ \\
\hline Anti-DNA Ab (IU/mL) & 1.5 & $<6.0$ \\
\hline Anti-RNP Ab (U/mL) & 2.3 & $<5.0$ \\
\hline Anti-Sm Ab (U/mL) & 1.9 & $<5.0$ \\
\hline Anti-SSA Ab (U/mL) & $<0.5$ & $<7.0$ \\
\hline Anti-SSB Ab (U/mL) & $<0.5$ & $<7.0$ \\
\hline Anti-cardiolipin (lgG) Ab (U/mL) & 2.3 & $<10$ \\
\hline Anti-cyclic citrullinated peptide $\mathrm{Ab}(\mathrm{U} / \mathrm{mL})$ & 1.2 & $<4.3$ \\
\hline Anti-topoisomerase Ab (U/mL) & 2.1 & $<7.0$ \\
\hline Anti-centromere Ab (index) & 4.1 & $<10.0$ \\
\hline Myeloperoxidase-ANCA (EU) & $<10$ & $<20$ \\
\hline PR3-ANCA (EU) & $<10$ & $<10$ \\
\hline Complement activity (U/mL) & 56 & $30-45$ \\
\hline $\mathrm{C} 3$ (mg/dL) & 164 & $68-128$ \\
\hline C4 (mg/dL) & 41 & $14-36$ \\
\hline Immune complexes-C1q ( $\mu \mathrm{g} / \mathrm{mL})$ & 1.8 & $<3.0$ \\
\hline Urine protein & $(-)$ & $(-)$ \\
\hline Occult blood in urine & $(-)$ & $(-)$ \\
\hline
\end{tabular}

Ig, immunoglobulin; Ab, antibody; ANCA, anti-neutrophil cytoplasmic antibodies. 

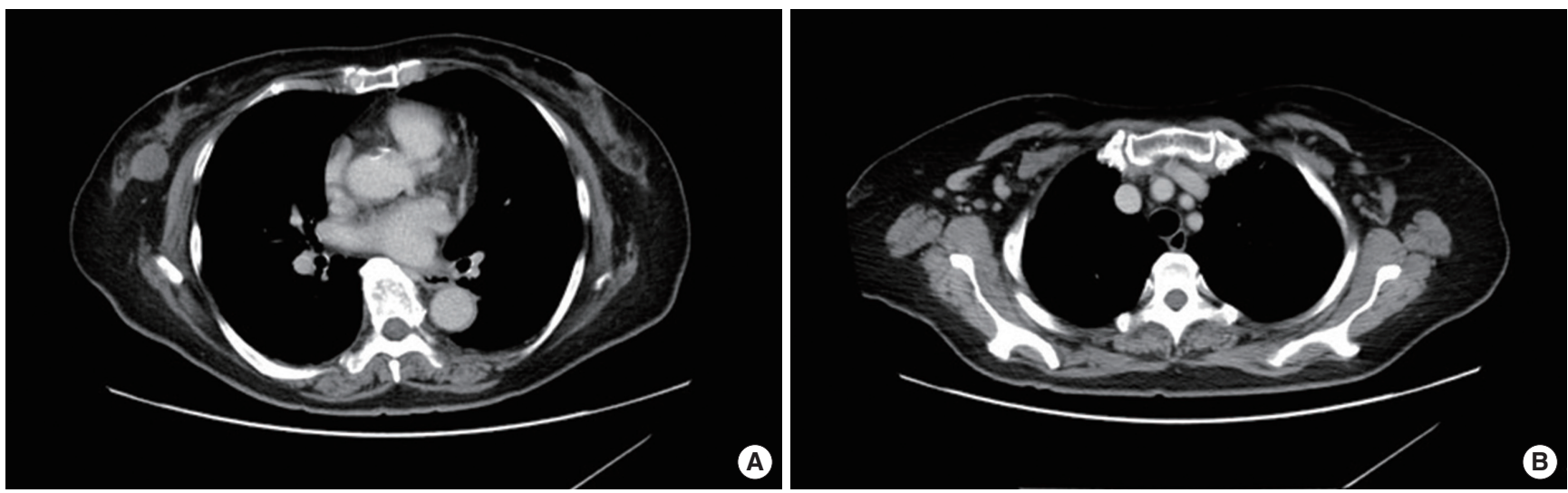

Fig. 2. (A) Chest computed tomography shows a mass shadow in the right breast. (B) Chest computed tomography shows bilateral axillary lymphadenopathy.

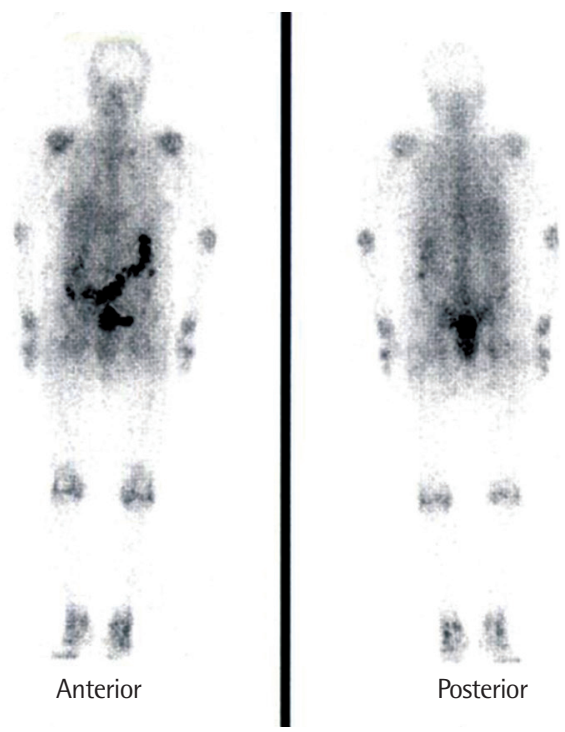

Fig. 3. Gallium scintigraphy shows uptake in the regions of bilateral shoulder, elbow, wrist, hand, knee, and foot joints.

anti-centromere, anti-topoisomerase-I, anti-cyclic citrullinated peptide antibody and myeloperoxidase and proteinase- 3 anti-neutrophil cytoplasmic antibodies. Urinalysis revealed neither proteinuria nor hematuria. Chest radiography and electrocardiography showed normal results. Ultrasonic cardiography revealed normal cardiac function. Bony destruction was not evident on articular Xray studies. Chest computed tomography (CT) revealed a mass in the right breast with bilateral axillary lymphadenopathy (Fig. 2A, B). Gallium scintigraphy revealed uptake in the regions of bilateral shoulder, elbow, wrist, hand, knee, and foot joints (Fig. 3). Esophagogastroduodenoscopy and colonoscopy showed no abnormal lesions associated with malignancy. Moreover, no malignant gynecological lesions were found. Ultrasonography-guided needle biop- sy of the right breast mass identified invasive ductal carcinoma. Both brain and abdominal CT revealed no abnormalities. Bone scintigraphy revealed no abnormal uptake. Based on these findings, RS3PE accompanied by breast carcinoma, a type of paraneoplastic syndrome, was diagnosed. Treatment was initiated with acetaminophen at $600 \mathrm{mg} /$ day and diclofenac sodium at $25 \mathrm{mg} /$ day for fever and arthralgia, but proved to have almost no effect (Fig. 4). For pitting edema, because the patient had suffered from uncontrolled DM and complained of great difficulty in grasping and walking due to the edema, she was treated with CAM at $800 \mathrm{mg} /$ day as an alternative to GC in expectation of its anti-inflammatory effects until surgical resection of the breast carcinoma could be performed. By about 10 days after starting CAM treatment, pitting edema showed considerable improvement (Fig. 1B). Fever and arthralgia improved slightly, with CRP level decreasing to $13.9 \mathrm{mg} /$ $\mathrm{dL}$. The patient underwent total mastectomy of the right breast and right axillary lymph node dissection (pT2N0M0). Histopathological examination of swollen axillary lymph nodes identified reactive lymphadenitis, so additional chemotherapy was considered unnecessary. Despite the resection of breast carcinoma, fever and arthralgia did not improve satisfactorily, with a CRP level of $9 \mathrm{mg} / \mathrm{dL}$. DM was largely under control; therefore, she was treated with prednisolone (PSL) at $10 \mathrm{mg} /$ day. Two weeks after starting PSL treatment, fever, arthralgia, and pitting edema had completely resolved (Fig. 1C), and CRP level had decreased to $0.2 \mathrm{mg} / \mathrm{dL}$.

\section{DISCUSSION}

The etiology of RS3PE remains unclear. However, RS3PE is sometimes associated with neoplastic and autoimmune diseases. Regard- 


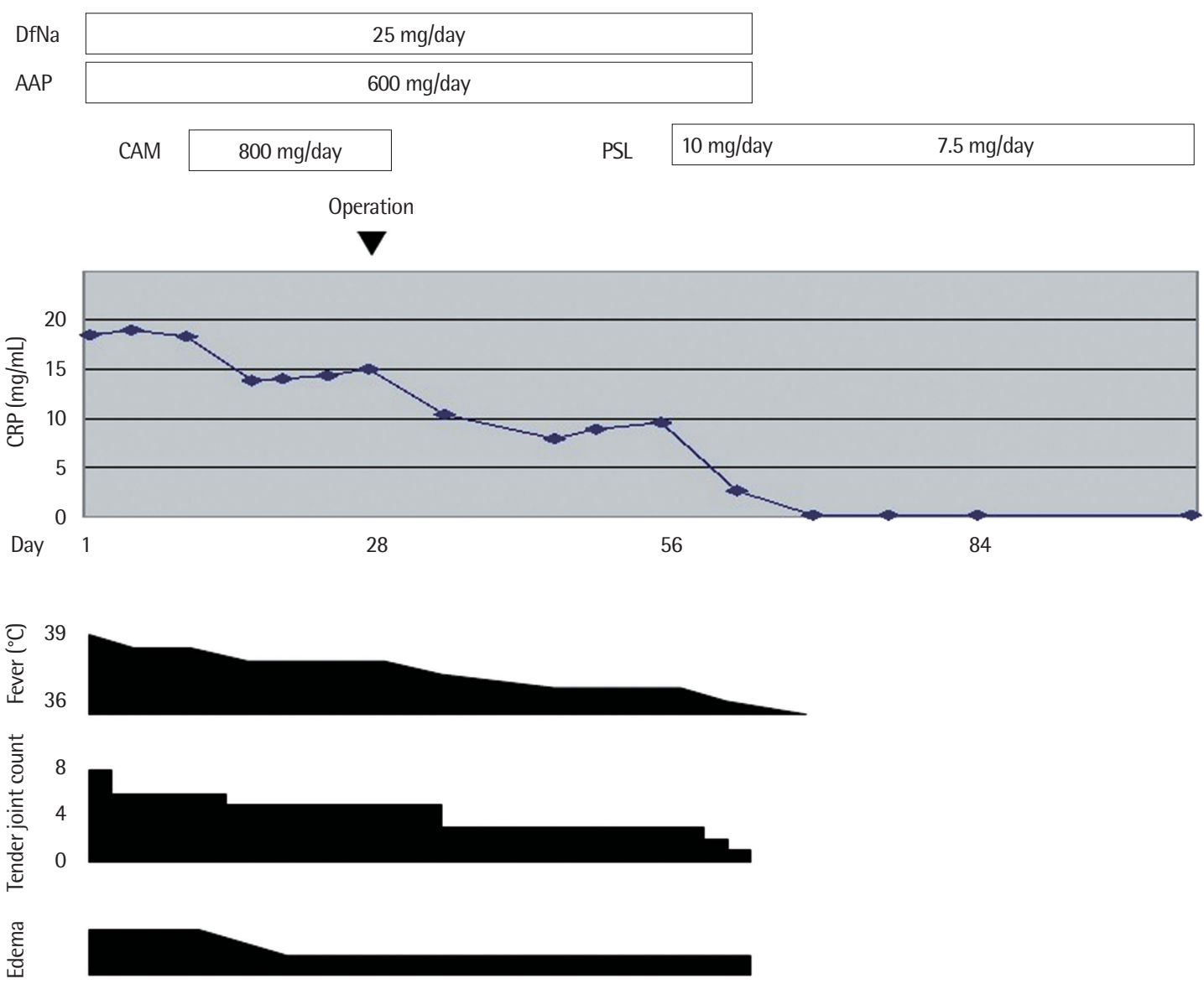

Fig. 4. Clinical course. AAP, acetaminophen; DfNa, diclofenac sodium; CAM, clarithromycin; PSL, prednisolone; CRP, C-reactive protein.

ing neoplastic disease, prostatic, gastric and colonic carcinomas seem to be the most frequently associated neoplasias [2]. Few cases of RS3PE associated with breast carcinoma have been reported [3]. The malignancy rate associated with RS3PE has been reported to be as high as $54 \%(32 / 59)$ [4]. The mechanism of association between RS3PE and neoplasma remains uncertain, but soluble factors such as interleukin (IL)-6 and tumor necrosis factor (TNF)- $a$ produced by the tumor may be involved [5]. Alternatively, proinflammatory cytokines including vascular endothelial growth factor (VEGF) triggered by neoplasia may be involved [4]. The synthesis of factors such as IL-6 is reportedly linked to paraneoplastic polyarthritis [6]. Similarly, elevated VEGF levels are reportedly associated with edema through the effects on permeability [7].

In most cases, symptoms of RS3PE associated with neoplastic disease disappear following surgical treatment of the carcinoma or chemotherapy. In the present case, however, clinical findings such as fever, arthralgia, and CRP level did not improve satisfactorily, despite resection of the carcinoma, but completely resolved with PSL. These findings may be attributable to breast carcinoma cells other than resected breast carcinoma cells remaining latent or some continued carcinoma-induced trigger on cytokine production. For RS3PE not associated with neoplastic disease, GC remains the mainstay of treatment. However, GC therapy is known to offer little efficacy against RS3PE associated with neoplastic disease. In the present case, GC therapy, namely PSL at $10 \mathrm{mg} /$ day, resulted in complete resolution of clinical findings within 2 weeks. Polyarthritis and edema with gastric carcinoma have been reported to be partially relieved by GC therapy [8]. GC therapy may thus prove effective against RE3PE associated with neoplastic disease, as in the present case.

CAM achieved considerable improvement of pitting edema, slight fever, arthralgia and elevated CRP levels. Apart from antibacterial activity, macrolides show a broad spectrum of pharmacological effects, including anti-inflammatory activity. Macrolides have been shown to affect several pathways of the inflammatory process, such as the production of proinflammatory cytokines [1]. In fact, CAM has been reported to suppress TNF- $\alpha$ and IL- 6 pro- 
duction [9]. Similarly, inhibitory effects of CAM on VEGF production have been recognized [10]. Based on these findings, the effectiveness of CAM against RS3PE might result from anti-inflammatory effects suppressing these cytokines, particularly VEGF.

RS3PE is one of the most common chronic inflammatory syndromes in elderly individuals. As elderly patients have a tendency to suffer from chronic diseases that are exacerbated by the use of GCs, such as DM, osteoporosis, and hypertension, CAM, particularly in combination with reduced conventional doses of GC, may offer a useful option for treating elderly patients with RS3PE. However, because only this single case has been reported, more research is necessary before CAM therapy can be recommended.

\section{REFERENCES}

1. Ogrendik M. Effect of clarithromycin in patients with active rheumatoid arthritis. Current Med Res Opin 2007;23:515-22.

2. Olivieri I, Salvarani C, Cantini F. RS3PE syndrome: an overview. Clin Exp
Rheumatol 2000;18:53-5.

3. Russell EB. Remitting seronegative symmetrical synovitis with pitting edema syndrome: follow up for neoplasia. J Rheumatol 2005;32:1760-1.

4. Yao Q, Su X, Altman RD. Is remitting seronegative symmetrical synovitis with pitting edema (RS3PE) a subset of rheumatoid arthritis? Semin Arthritis Rheum 2010;40:89-94.

5. Nagasawa K. Rheumatic manifestations in paraneoplastic syndrome. Intern Med 2000;39:685-6.

6. Sibilia J, Friess S, SchaeverbekeT, Maloisel F, Bertin P, GoichotB, et al. Remitting seronegative symmetrical synovitis with pitting edema (RS3PE): a form of paraneoplastic polyarthritis? J Rheumatol 1999;26:115-20.

7. Arima K, Origuchi T, Tamai M, Iwanaga N, Izumi Y, Huang M, et al. RS3PE syndrome presenting as vascular endothelial growth factor associated disorder. Ann Rheum Dis 2005;64:1653-5.

8. Tada Y, Sato H, Yoshizawa S, Kimura H, Kitamura M, Kusaba T, et al. Remitting seronegative symmetrical synovitis with pitting edema associated with gastric carcinoma. J Rheumatol 1997;24:974-5.

9. Khan AA, Slifer TR, Araujo FG, Remington JS. Effect of clarithromycin and azithromycin on production of cytokines by human monocytes. Int J Antimicrob Agents 1999;11:121-32.

10. Matsune S, Sun D, Ohori J, Nishimoto K, Fukuiwa T, Ushikai M, et al. Inhibition of vascular endothelial growth factor by macrolides in cultured fibroblasts from nasal polyps. Laryngoscope 2005;115:1953-6. 